\title{
Estudo da demanda de uma equipe da Estratégia Saúde da Família que utiliza o acesso avançado como modelo de organização da agenda
}

Study of the demand for a Family Health Strategy (FHS) team that uses advanced access (AA) as a model for organizing the agenda

\section{Estudio de la demanda de un equipo de Estrategia de Salud de la Familia que utilice el acceso avanzado como modelo para la organización de la agenda}

\author{
Liliane Ecco Canuto ${ }^{1 \oplus}$, Angela Fernandes Leal da Silva ${ }^{\circledR}$, Luiza Sanchez Palacio Pinheiro ${ }^{2 \oplus}$, Júlio Cesar Arruda Canuto Júnior ${ }^{1 \oplus}$, \\ Nereida Lúcia Palko dos Santos² \\ ${ }^{1}$ Secretaria Municipal de Saúde/RJ \\ ${ }^{2}$ Universidade Federal do Rio de Janeiro
}

\section{Resumo}

Introdução: $O$ acesso oportuno e a continuidade do cuidado são duas medidas operacionais importantes para a prática dos cuidados na Atenção Primária à Saúde (APS). Objetivo: Realizar um estudo da demanda de uma equipe da Estratégia Saúde da Família (ESF) que utiliza o acesso avançado (AA) como modelo de organização da agenda. Métodos: Trata-se de uma análise documental, quantitativa, descritiva e retrospectiva realizada em uma equipe da ESF no Rio de Janeiro. Os 1410 dados foram coletados em uma planilha do Microsoft ${ }^{\circledR}$ Excel $^{\circledR}$ no Google Drive, utilizada no processo de trabalho da equipe e foram tabulados e analisados em planilhas do Microsoft Excel ${ }^{\circledR}$. Foram incluídos os registros do período de agosto e setembro de 2017 e excluídos os registros no qual o motivo da consulta (MC) não foi preenchido. Os MC foram codificados a partir de demandas, queixas e necessidades expressas nas narrativas dos usuários, e adaptadas à conformidade da codificação da CIAP (Classificação Internacional de Atenção Primária). Resultados: Dos 1392 registros de $M C$ dos usuários restantes, 149 não foram atendidos por motivo de falta, reagendamento ou evasão, totalizando 1243 registros analisados. Predominou o sexo feminino, faixa etária entre 55 e 59 anos, atendimentos realizados em demanda espontânea, pela manhã, principalmente nas primeiras horas do acolhimento e com queixas gerais e não-específicas. Conclusão: Com o AA parece haver uma acessibilidade maior aos usuários que poderão ter o acesso de primeiro contato mais facilmente para a resolução dos seus problemas. Realizar o estudo da demanda possibilita o levantamento de dados para a elaboração de um planejamento para uma melhor organização da agenda refletindo em um melhor atendimento à população de forma resolutiva, oportuna e integral.

Palavras-chave: Atenção Primária à Saúde; Estratégia Saúde da Família; Acesso aos Serviços de Saúde; Agendamento de Consultas; Classificação Internacional de Doenças.

\author{
Autor correspondente: \\ Liliane Ecco Canuto. \\ E-mail: lilianeecco@gmail.com \\ Fonte de financiamento: \\ não se aplica. \\ Parecer CEP: \\ Secretaria Municipal de Saúde do Rio de \\ Janeiro - SMS/RJ (Parecer: 2.850.224) e \\ Hospital Escola São Francisco de Assis da \\ UFRJ/EEAN (Parecer: 2.790.170) \\ Procedência: \\ não encomendado. \\ Recebido em: 11/02/2020. \\ Aprovado em: 10/12/2020. \\ Editor Associado: \\ Claudio Claudino da Silva Filho
}




\begin{abstract}
Introduction: Timely access and continuity of care are two important operational measures for the practice of care in Primary Health Care (PHC). Purpose: Conduct a study of the demand for a Family Health Strategy (FHS) team that uses advanced access (AA) as a model for organizing the schedule. Methods: It is a documentary, quantitative, descriptive and retrospective analysis carried out in an FHS team in Rio de Janeiro. The 1410 pieces of data were collected in a Microsoft ${ }^{\circledR}$ Excel $^{\circledR}$ spreadsheet in Google Drive, used in the team's work process and were tabulated and arranged in Microsoft Exce ${ }^{\circledR}$ spreadsheets. Records from August and September 2017 were included, while records in which the reason for the consultation $(\mathrm{RC})$ was not filled out were excluded. The $\mathrm{RC}$ were coded based on the demands, complaints and needs expressed in the users' narratives, and adapted to the conformity of the ICPC (International Classification of Primary Care). Results: Of the 1392 RC records of the remaining users, 149 were not attended due to absence, rescheduling or evasion, totaling 1243 records covered. There was a predominance of the following variables: females, aged between 55 and 59 years old, spontaneous demand queries, visits in the morning, mainly in the first hours of the reception and about general and non-specific complaints. Conclusions: With AA there seems to be greater accessibility for users who receive first contact access more easily to solve their problems. Carrying out the demand study makes it possible to collect data for the elaboration of a plan for a better organization of the agenda, reflecting in a better service to the population in a resolute, timely and integral way.
\end{abstract}

Keywords: Primary Health Care, Family Health Strategy, Health Services Accessibility, Appointments and Schedules, International Classification of Primary Care.

\title{
Resumen
}

Introducción: El acceso oportuno y la continuidad de la atención son dos importantes medidas operativas para la práctica de la atención en Atención Primaria de Salud (APS). Objetivo: Realizar un estudio de demanda de un equipo de Estrategia de Salud de la Familia (ESF) que utilice el acceso avanzado (AA) como modelo para la organización de la agenda. Métodos: se trata de un análisis documental, cuantitativo, descriptivo y retrospectivo realizado en un equipo de la ESF en Río de Janeiro. Los datos 1410 se recopilaron en una hoja de cálculo de Microsoft ${ }^{\circledR}$ Excel ${ }^{\circledR}$ en Google Drive, se utilizaron en el proceso de trabajo del equipo y se tabularon y analizaron en hojas de cálculo de Microsoft Excel ${ }^{\circledR}$. Se incluyeron registros del período de agosto y septiembre de 2017 y se excluyeron los registros en los que no se llenó el motivo de la consulta (MC). Los MC fueron codificados en base a las demandas, quejas y necesidades expresadas en las narrativas de los usuarios, y se adaptaron a la conformidad de la codificación CIAP (Clasificación Internacional de Atención Primaria). Resultados: De los 1392 registros de MC del resto de usuarios, 149 no fueron atendidos por inasistencia, reprogramación o evasión, totalizando 1243 registros analizados. Predominó el sexo femenino, con edades comprendidas entre los 55 y los 59 años, visitas realizadas en demanda espontánea, por la mañana, principalmente en las primeras horas de la recepción y con quejas generales y no específicas. Conclusión: Con AA parece haber una mayor accesibilidad para los usuarios que podrán tener acceso de primer contacto más fácilmente para resolver sus problemas. La realización del estudio de demanda permite recabar datos para la elaboración de un plan para una mejor organización de la agenda, reflejándose en un mejor servicio a la población de manera resuelta, oportuna e integral.

Palabras clave: Atención Primaria de Salud, Estrategia de Salud Familiar, Accesibilidad a los Servicios de Salud, Citas y Horarios, Clasificación Internacional de la Atención Primaria.

\section{INTRODUÇÃO}

A Estratégia Saúde da Família (ESF) visa à reorganização da Atenção Primária à Saúde (APS) no País, de acordo com os preceitos do Sistema Único de Saúde (SUS), e é tida como estratégia de expansão, qualificação e consolidação da APS. Ela favorece uma reorientação do processo de trabalho com maior potencial de aprofundar os princípios, diretrizes e fundamentos da APS, de ampliar a resolutividade e impacto na situação de saúde das pessoas e coletividades, além de propiciar uma importante relação custo-efetividade ${ }^{1}$.

No SUS, a ESF, representa o principal modelo para a organização da APS e o seu fortalecimento é estruturante para o estabelecimento das Redes de Atenção à Saúde (RAS), que integradas por meio de sistemas de apoio técnico, logístico e de gestão, buscam garantir a integralidade do cuidado ${ }^{2}$. A APS, além de ser o centro de comunicação das RAS, é a principal porta de entrada do sistema, integradora, coordenadora do cuidado e ordenadora das ações e serviços disponibilizados na rede, servindo como base para a efetivação da integralidade e cumprindo do papel estratégico na rede de atenção'. 
Entre os modelos existentes para a abordagem da demanda que podem ser utilizados na APS, estão o modelo tradicional - conheça a demanda urgente agora e conheça a demanda não urgente mais tarde -, o modelo Carve Out - prever a demanda urgente e reservar tempo para conhecê-la - e o modelo de acesso avançado (AA) - fazer o trabalho de hoje, hoje. Nesse último modelo, não há a classificação da demanda em rotina e urgência. Os usuários que procuram a sua equipe nas unidades da APS, são agendados para o mesmo dia $^{3}$, onde aproximadamente $65 \%$ das vagas da agenda estão disponíveis para o atendimento no mesmo dia, e $35 \%$ estão reservadas para agendamentos ${ }^{4}$.

O acesso oportuno e a continuidade do cuidado são duas medidas operacionais importantes para a prática dos cuidados na $\mathrm{APS}^{5}$. Nela, o acesso se dá de maneira universal, independente do grau de necessidade, uma vez que não se pode esperar que os indivíduos conheçam a gravidade ou urgência de muitos dos seus problemas ao procurarem atendimento. Se a acessibilidade é comprometida, a atenção pode ser postergada a ponto de afetar o diagnóstico e o manejo do problema ${ }^{6}$. É necessário planejar para atingir metas e objetivos, de modo a dispensar a sorte e as circunstâncias para definir aonde se quer chegar ${ }^{7}$.

O AA parece ser o mais promissor ao conseguir equilibrar a capacidade de oferta das equipes e serviços de saúde e as demandas de usuários e famílias, sendo capaz de reduzir simultaneamente o tempo de espera. Entretanto, só é possível modificar o sistema de agendamento na presença de uma estrutura disponível para sustentá-lo ${ }^{8}$. Como em outras intervenções, para a sua implementação deve-se considerar as características da população e do ambiente de prática ${ }^{9}$. Além disso, após a implementação de um processo de organização do trabalho e do acesso tão abrangente como o AA, há a necessidade de analisar os seus efeitos ${ }^{10}$.

Nesse contexto, é no cenário da APS, em uma equipe de saúde de uma Clínica da Família (CF) do município do Rio de Janeiro, que o AA ganha destaque, como modelo mais adotado de organização da agenda, desde 2015, com a redução do tempo de espera para atendimento como elemento fundamental ao acesso, assim como a eficácia na assistência à saúde. Entretanto, até o presente momento, não foram realizados estudos da demanda com foco no planejamento e organização da agenda desta equipe, podendo influenciar e/ou relacionar ao maior tempo de espera dos usuários no dia da consulta e a ocorrência de evasões no atendimento ${ }^{11}$.

Sendo assim, diante da observação empírica do cotidiano do acesso dos usuários, evidenciouse a necessidade por um estudo da demanda para subsidiar e compor elementos ao planejamento e programação do processo de trabalho da equipe de saúde, uma vez que, o primeiro passo para que seja realizado esse planejamento implica em conhecer o território e a realidade local, por meio da análise da situação de saúde, envolvendo o diagnóstico da comunidade e o estudo da demanda ${ }^{12}$.

Diante do exposto, o objetivo desse estudo é realizar um estudo da demanda de uma equipe da ESF que utiliza o AA como modelo de organização da agenda.

\section{MÉTODOS}

Trata-se de uma análise documental, quantitativa, descritiva e retrospectiva realizada nos registros diários do acesso avançado realizado em planilha do Microsoft ${ }^{\circledR}$ Exce $^{\circledR}$ no Google Drive, utilizada pela equipe de saúde da ESF em uma CF da área programática 3.1 do município do Rio de Janeiro, como 
método de registro formalizado do processo de trabalho local e como forma de ordenação dos atendimentos e classificação de risco.

A CF foi inaugurada em abril de 2010 e na época do estudo contava com 15 equipes de saúde da família que atendiam aproximadamente 46.000 pessoas, sendo a equipe escolhida para o estudo, responsável por aproximadamente 3.000 pessoas. O horário de funcionamento da CF no período, era de segunda à sexta-feira das 07 às 19 horas e no sábado das 08 às 12 horas.

No período de registro dos dados coletados, de agosto a setembro de 2017, a equipe contava com cinco Agentes Comunitários de Saúde (ACS), sendo cada um deles responsável por uma micro área, ou seja, o espaço geográfico correspondente a sua área de atuação. Também contava com dois residentes e um preceptor de Medicina de Família e Comunidade (MFC), duas residentes e uma preceptora de Enfermagem em Saúde da Família, uma técnica de enfermagem, uma dentista e uma auxiliar de saúde bucal responsáveis por duas equipes.

A residente do segundo ano de enfermagem encontrava-se realizando estágios externos. Na quarta à tarde a equipe se reunia para a reunião de equipe, atendendo apenas usuários agendados após a reunião ou que não poderiam retornar em outro momento. Os residentes ficavam à frente da equipe, sendo os profissionais responsáveis pelos atendimentos, portanto a agenda fixa de consultas não contemplava os preceptores, que tinham a função de fazer suporte "ombro a ombro" e, em turnos de maior demanda, auxiliar nos atendimentos excedentes atendendo parte desses pacientes em outro consultório. Uma organização padrão, porém variável, da grade semanal da equipe está representada no quadro 1.

A planilha usada no acolhimento era dividida em dias e turnos de atendimento. Durante a semana, o acolhimento do turno da manhã funcionava das 08 às 12:30 horas e no turno da tarde das 12:30 às 17 horas. No horário das 17 às 19 horas eram atendidos os usuários que foram acolhidos durante o dia e ainda aguardavam por atendimento. Nesse horário, como a chegada de usuários na clínica era reduzida, eram escalados dois ACS por dia para o acolhimento de todas as 15 equipes. Na ocorrência da chegada de algum usuário, os ACS se comunicavam verbalmente com os profissionais em atendimento. Aos sábados o acolhimento funcionava das 08 às 12:00 horas. Nesse dia, a procura por atendimento era reduzida e buscava-se a longitudinalidade com os usuários sendo atendidos por demandas clínicas não agudas pela sua equipe de referência, caso houvesse disponibilidade, durante a semana. Dessa forma, era escalada uma equipe para trabalhar em cada sábado. No período da coleta de dados a equipe em estudo trabalhou em apenas um sábado.

Quadro 1. Organização padrão da grade semanal dos profissionais da equipe.

\begin{tabular}{|c|c|c|c|c|c|c|}
\hline & & Segunda & Terça & Quarta & Quinta & Sexta \\
\hline \multirow{2}{*}{$\frac{\sqrt{\frac{\pi}{c}}}{\frac{5}{\pi}}$} & Med & $\begin{array}{l}\text { R1: Atendimento } \\
\text { R2: Atividades } \\
\text { administrativas }\end{array}$ & $\begin{array}{l}\text { R1: Atendimento } \\
\text { R2: Plantão }\end{array}$ & $\begin{array}{l}\text { R1: Atendimento } \\
\text { R2: Atendimento }\end{array}$ & $\begin{array}{c}\text { R1: VD } \\
\text { R2: Atendimento }\end{array}$ & $\begin{array}{l}\text { R1: Atendimento } \\
\text { R2: Atendimento }\end{array}$ \\
\hline & Enf & R1: Aula & R1: VD & R1: Estudo clínico & R1: Atendimento & R1: Atendimento \\
\hline \multirow[t]{2}{*}{$\frac{0}{\frac{0}{0}}$} & Med & $\begin{array}{l}\text { R2: Atendimento } \\
\text { R2: Atividades } \\
\text { administrativas }\end{array}$ & $\begin{array}{c}\text { R1: Aula } \\
\text { R2: Plantão }\end{array}$ & Reunião de equipe & R1: Atendimento & $\begin{array}{l}\text { R1: Atendimento } \\
\text { R2: Atendimento }\end{array}$ \\
\hline & Enf & R1: Aula & R1: Atendimento & Reunião de equipe & R1: Atendimento & R1: Atendimento \\
\hline
\end{tabular}


Nos horários previamente expostos de atendimento no acolhimento, os ACS preenchiam informações básicas do usuário como o nome e a idade e descreviam qual o motivo da consulta (MC) que o usuário relatou naquele momento. Após esse relato, o médico e o enfermeiro decidiam qual profissional iria atendê-lo e registravam na mesma planilha. Essa decisão era determinada pelos próprios profissionais que acordavam previamente as principais demandas responsáveis por cada categoria e revisavam sempre que necessário nas reuniões de equipe. Em caso de dúvida, os profissionais conversavam por mensagem na planilha, em tempo real, sobre quem realizaria o atendimento. Também era possível sinalizar quando o usuário deveria ser encaminhado para outro setor como atendimento odontológico, por exemplo. Na planilha também eram identificados os usuários que estavam agendados em demanda programada, por turno.

Os dados coletados foram tabulados em planilhas do software Microsoft Excel ${ }^{\circledR}$ e analisados através de frequência simples, média, mediana e através do recurso de Tabela Dinâmica do mesmo software. A unidade de análise central da planilha para o estudo, foram os $\mathrm{MC}$, assim, foram incluídos os dados registrados na planilha de atendimento da equipe que continham a $\mathrm{MC}$, e excluídos registros no qual o MC não foi preenchido.

Para efeitos deste trabalho, os MC descritos pelos ACS a partir das demandas, queixas e necessidades expressas nas narrativas dos usuários foram analisados pelo pesquisador e codificados por esse para a CIAP (Classificação Internacional de Atenção Primária). A CIAP classifica todos os capítulos com base nos sistemas anatômicos, partindo do princípio de que a localização (ou a pessoa) tem primazia sobre a etiologia, dividida em 17 capítulos e 7 componentes ${ }^{13}$.

O projeto foi submetido à Plataforma Brasil e aprovado pelo Comitê de Ética em Pesquisa da Secretaria Municipal de Saúde do Rio de Janeiro - SMS/RJ (Parecer: 2.850.224) e UFRJ - Escola de Enfermagem Anna Nery - Hospital Escola São Francisco de Assis da Universidade Federal do Rio de Janeiro / EEAN (Parecer: 2.790.170), com o objetivo de cumprir o disposto na Resolução $n^{\circ} 466 / 12$ do Conselho Nacional de Saúde.

\section{RESULTADOS}

No período de agosto e setembro de 2017, foram efetuados 1.410 registros na planilha. Desses, 17 foram excluídos por não apresentarem o MC. Dos 1.392 registros de MC dos usuários restantes, 149 $(10,7 \%)$ não foram atendidos, sendo $71(5,1 \%)$ por falta, 39 (2,8\%) por reagendamento assim como 39 $(2,8 \%)$ por evasão, totalizando 1.243 registros analisados.

Entre os atendimentos realizados, o sexo predominante foi o feminino com 820 (65,9\%) atendimentos. A idade dos usuários variou de 0 a 96 anos com média de 38,3 e mediana de 38 anos. A faixa etária predominante foi a de 55 a 59 anos com 118 atendimentos (9,5\%), seguida de menor ou igual a dois anos com 103 atendimentos (8,3\%).

Entre os atendimentos dessa última faixa etária, 52 (50,5\%) foram consultas de puericultura. Proporcionalmente, os meninos tiveram mais atendimentos de puericultura com 35 (54\%) dos 64 atendimentos que as meninas com 17 (43\%) dos 39 atendimentos, enquanto que as meninas tiveram mais faltas nas consultas de puericultura que os meninos, com $20(57,1 \%)$ e $15(42,9 \%)$ faltas respectivamente e foram proporcionamente mais atendidas em demanda espontânea com 22 dos 39 (57\%) atendimentos 
do que os meninos, que tiveram 29 dos 64 (46\%) atendimentos. Entre os atendimentos realizados em demanda espontânea nessa faixa etária, predominaram os relacionados a pele e ao aparelho respiratório com $15(42,9 \%)$ e $13(37,1 \%)$ atendimentos respectivamente.

Com relação ao turno de atendimento, foram avaliados 78 turnos e houve predomínio do turno matutino com $61,5 \%$. Os 764 atendimentos matutinos foram realizados em 44 turnos, sendo desses, 30 com dois profissionais (medicina e enfermagem) e 14 com apenas um profissional em atendimento, com uma média de 17,3 atendimentos por manhã. Os 479 atendimentos vespertinos foram realizados em 34 turnos, sendo desses, 21 com dois profissionais e 13 com um profissional em atendimento, com uma média de 14 atendimentos por turno.

O dia da semana com o maior número de atendimentos foi a quinta-feira (284) e a quarta-feira, na parte da manhã, concentrou o maior número de atendimentos (173) quando divididos por turnos conforme apresentado no gráfico 1.

Do total de atendimentos 944 (75,9\%) foram realizados pelo médico (com média de 12,7 atendimentos por turno) 289 (23,3\%) pelo enfermeiro (com média de 6,5 atendimentos por turno) e $10(0,8 \%)$ por ambos os profissionais. Houve $27(2,2 \%)$ atendimentos de usuários de outras equipes e $8(0,6 \%)$ de outras CF de forma pactuada quando essas estavam sem profissional da equipe de referência para atendimento da demanda aguda.

Com relação ao tipo de demanda, 1.075 (86,5\%) dos atendimentos realizados foram em demanda espontânea e 168 (13,5\%) em demanda programada. Dos agendamentos realizados, 71 (29,1\%) usuários não compareceram em consulta e $41(16,8 \%)$ chegaram com mais de 15 minutos de atraso. Entre os faltosos, a maioria era de consultas de puericultura com $36(50,7 \%)$ do total.

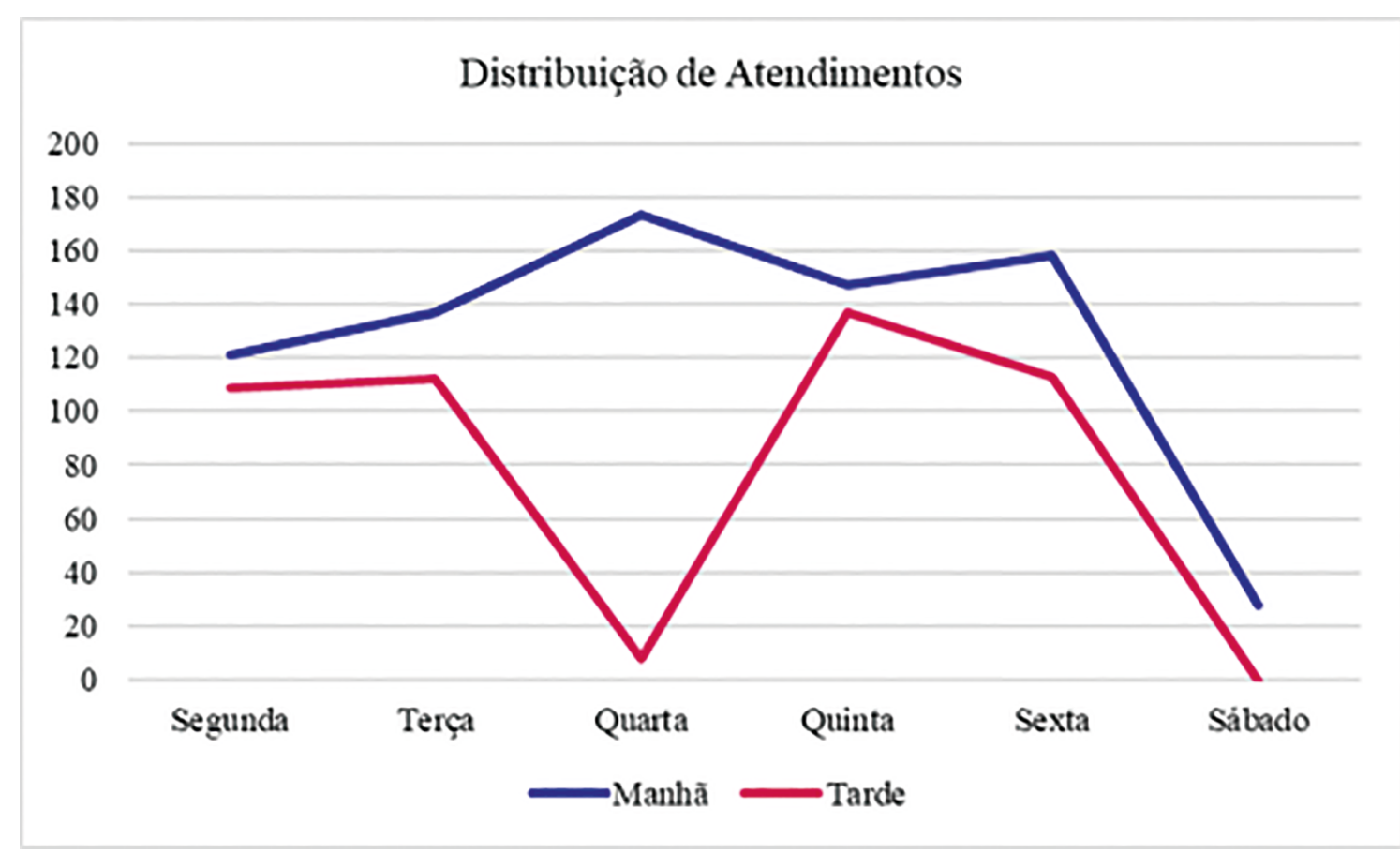

Gráfico 1. Distribuição dos atendimentos por dia da semana e turno. 
Dos usuários atendidos em demanda espontânea no turno da manhã, 378 (54,5\%) chegaram na CF até às 09 horas e $533(76,9 \%)$ até às 10h. Na parte da tarde, $148(38,7 \%)$ chegaram até às 14 horas e $266(69,6 \%)$ até às 15 horas.

A quantidade de $\mathrm{MC}$ relatados por usuários nos acolhimentos foi de um para $1.083(87,1 \%)$, dois para $136(10,9 \%)$ e três ou mais para $24(1,9 \%)$ dos usuários. No período da pesquisa, $607(74,2 \%)$ usuários que buscaram atendimento em demanda espontânea foram atendidos uma vez, $136(16,6 \%)$ duas vezes, $52(6,4 \%)$ três vezes, $17(2,1 \%)$ quatro vezes, $4(0,5 \%)$ cinco vezes e $2(0,2 \%)$ seis vezes.

De acordo com a CIAP, o componente mais frequente foi o componente 1- Queixas e sintomas, responsável por $624(50,2 \%)$ atendimentos, seguido pelo componente 7- Diagnósticos e doenças com 205 (16,5\%) e 4- Resultados de exames com 196 (15,8\%) atendimentos.

Entre o sexo feminino também houve prevalência do capítulo $A(21,6 \%)$, seguido do capítulo $X$ $(14,4 \%)$ e W $(12,4 \%)$, enquanto entre o sexo masculino, houve predomínio do capítulo $A(32,2 \%)$, seguido do capítulo $\mathrm{K}(14,4 \%)$ e S $(11,3 \%)$ (Tabela 1$)$.

Entre os atendimentos realizados pelo médico, houve prevalência do capítulo $A(26,0 \%)$, seguido dos capítulos $\mathrm{K}(10,1 \%)$ e $\mathrm{P}(9,6 \%)$ (Tabela 2). Entre os atendimentos realizados pela enfermagem, prevaleceram os capítulos X (29,3\%), seguido dos capítulos A $(26,5 \%)$ e K (20,5\%) (Tabela 2). A enfermagem realizou atendimentos classificados de acordo com o MC relacionados à 14 dos 17 capítulos da CIAP e com 61 códigos diferentes.

Entre os MC mais frequentes de acordo com o que foi expresso pelo usuário e classificado pela CIAP estão: "A60- Resultados de análises/procedimentos do capítulo geral e não-específico", "K86- Hipertensão sem complicações" e "W78- Gravidez".

Tabela 1. Distribuição dos MC de acordo com o sexo segundo os capítulos da CIAP.

\begin{tabular}{|c|c|c|c|c|}
\hline Capítulos & \multicolumn{2}{|c|}{ Feminino } & \multicolumn{2}{|c|}{ Masculino } \\
\hline A- Geral não específico & 177 & 21,6 & 136 & 32,2 \\
\hline D- Aparelho digestivo & 36 & 4,4 & 21 & 5,0 \\
\hline F-Olhos & 15 & 1,8 & 10 & 2,4 \\
\hline K- Aparelho circulatório & 85 & 10,4 & 61 & 14,4 \\
\hline L- Sistema musculoesquelético & 52 & 6,3 & 29 & 6,9 \\
\hline $\mathrm{N}$ - Sistema nervoso & 38 & 4,6 & 12 & 2,8 \\
\hline P- Psicológico & 72 & 8,8 & 23 & 5,4 \\
\hline R-Aparelho respiratório & 52 & 6,3 & 35 & 8,3 \\
\hline W- Gravidez e planejamento familiar & 102 & 12,4 & - & - \\
\hline X-Aparelho genital feminino & 118 & 14,4 & - & - \\
\hline Y- Aparelho genital masculino & - & - & 8 & 1,9 \\
\hline Total & 820 & 100,0 & 423 & 100,0 \\
\hline
\end{tabular}


Tabela 2. Distribuição dos MC com atendimento realizado pelos profissionais enfermeiro e médico de acordo com os capítulos da CIAP.

\begin{tabular}{|c|c|c|c|c|}
\hline \multirow{2}{*}{ Capítulos } & \multicolumn{2}{|c|}{ Enfermeiro } & \multicolumn{2}{|c|}{ Médico } \\
\hline & $\mathbf{n}$ & $\%$ & $\mathbf{n}$ & $\%$ \\
\hline A-Geral não específico & 73 & 29,3 & 245 & 26,0 \\
\hline B- Sangue, órgãos hematopoiéticos e linfáticos & 1 & 0,4 & 8 & 0,8 \\
\hline D- Aparelho digestivo & 5 & 2,0 & 52 & 5,5 \\
\hline F-Olhos & - & - & 25 & 2,6 \\
\hline H- Ouvidos & - & - & 19 & 2,0 \\
\hline K- Aparelho circulatório & 51 & 20,5 & 95 & 10,1 \\
\hline L- Sistema musculoesquelético & 4 & 1,6 & 76 & 8,1 \\
\hline N- Sistema nervoso & 6 & 2,4 & 43 & 4,6 \\
\hline P- Psicológico & 3 & 1,2 & 91 & 9,6 \\
\hline R- Aparelho respiratório & 15 & 6,0 & 70 & 7,4 \\
\hline S- Pele & 13 & 5,2 & 84 & 8,9 \\
\hline T- Endócrino, metabólico e nutricional & 4 & 1,6 & 15 & 1,6 \\
\hline U- Aparelho urinário & 3 & 0,8 & 12 & 1,3 \\
\hline W- Gravidez e planejamento familiar & 43 & 17,3 & 59 & 6,3 \\
\hline X-Aparelho genital feminino & 73 & 29,3 & 44 & 4,7 \\
\hline Y- Aparelho genital masculino & 2 & 0,8 & 6 & 0,6 \\
\hline TOTAL & 249 & 100,0 & 944 & 100,0 \\
\hline
\end{tabular}

Ao dividir os MC de acordo com o sexo, em ambos o principal motivo foi "A60- Resultado de Análises/ Procedimentos (capítulo Geral e não-específico)". No sexo feminino, o principal MC foi seguido por "W78Gravidez" e "X37- Citologia esfoliativa/histologia". No sexo masculino, o principal motivo foi seguido de "K86- Hipertensão sem complicações" e "A98- Medicina Preventiva/Manutenção da Saúde”. Os principais MC de acordo com o sexo estão apresentados no gráfico 2.

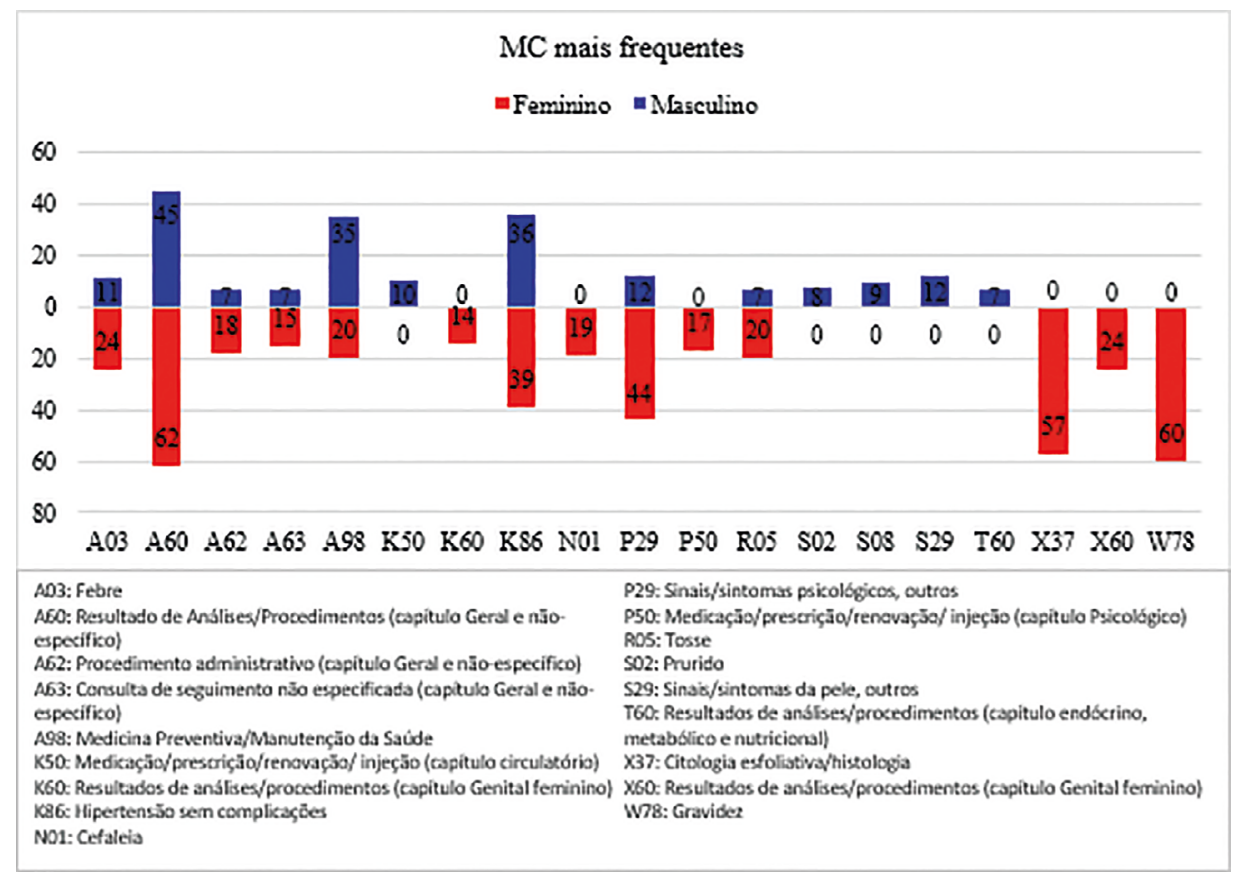

Gráfico 2. Motivos de consulta mais frequentes em uma Equipe da Estratégia Saúde da Família, de acordo com o sexo, codificados pela CIAP. 


\section{DISCUSSÃO}

A maioria da população cadastrada na equipe é do sexo feminino (53,6\%). Essa diferença é maior quando analisamos a diferença entre os sexos no acesso ao serviço, que se deu majoritariamente por mulheres, como pode ser observado no gráfico 3. Analisando o mesmo gráfico, fica evidente como os homens em idade adulta acessam menos o serviço de saúde. A maior utilização dos serviços de saúde da APS pelo sexo feminino já é bem estabelecida na literatura ${ }^{14,15,16,17}$, assim como os dados encontrados em uma revisão bibliográfica ${ }^{18}$ que afirmam que crianças, mulheres e idosos acessam mais os serviços de saúde. Isso também pode ser observado ao analisarmos a pirâmide etária da população que acessou o serviço no nosso estudo.

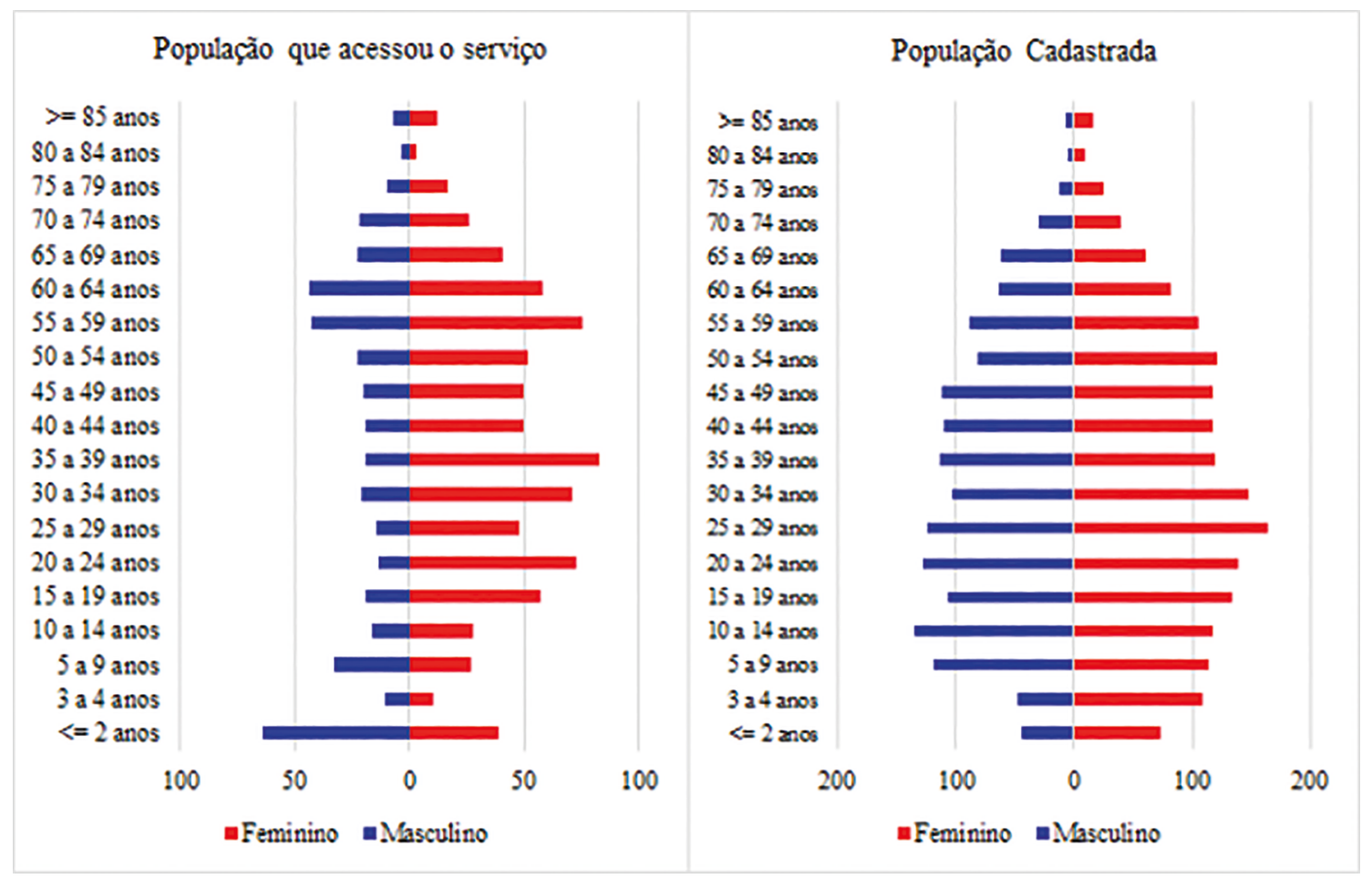

Gráfico 3. Distribuição da população que acessou o serviço e da população cadastrada por faixa etária e sexo.

Os homens procuram menos os serviços de saúde, principalmente os de caráter preventivo. As justificativas envolvem aspectos culturais ${ }^{15}$ como a masculinidade e a invulnerabilidade deste gênero, aspectos laborais que envolvem a carga horária no trabalho, ausência de atestados que poderiam ter sido emitidos por profissionais, filas e horários de atendimento incompatíveis e a invisibilidade dos homens na $\mathrm{APS}^{19}$.

É importante a reorganização, a resolutividade e a implementação de estratégias dos serviços de saúde para sensibilizar a população masculina que concentra grande parte da não adesão aos serviços de saúde com estratégias como as apresentadas na Política Nacional de Atenção Integral à Saúde do Homem ${ }^{18}$ a fim de garantir o acesso ao serviço de saúde por essa população ${ }^{19}$.

Pode-se observar que apesar de o número de crianças menores de 2 anos cadastradas ser maior no sexo feminino, houve um número maior de consultas no sexo masculino, sendo necessário uma maior 
investigação para a exploração desse dado. Outro dado interessante foi a proporção de atendimentos realizados em demanda espontânea e puericultura por sexo: enquanto que os meninos tiveram mais atendimentos de puericultura que em demanda espontânea, as meninas, de forma inversamente porporcional, tiveram mais atendimentos em demanda espontânea que de puericultura. Na ESF a puericultura vem se mostrando como uma excelente ferramenta de promoção da saúde e redução de morbimortalidade e a ausência nas consultas pode levar às crianças a situações de risco, sendo importante investigar os motivos para o não comparecimento ${ }^{20}$.

Também pode-se observar um número considerado de consultas em crianças, relacionados a problemas respiratórios e de pele, assim como em outro estudo realizado em Minas Gerais ${ }^{15}$. Esse dado reforça o fato de que a ESF deve investir em orientações para a prevenção desses agravos e de suas complicações nessa população promovendo estratégias de apoio às famílias para a prevenção de internações ${ }^{21}$.

Com relação a distribuição dos atendimentos, é importante observar que apesar de ser uma equipe de residência que está em processo de formação e aprimoramento de seus atendimentos e técnicas, e que além dos atendimentos os residentes desenvolviam outras atividades fora do consultório, pode-se observar que quando ambos os profissionais estavam atendendo ao mesmo tempo, a produtividade da equipe aumenta em aproximadamente $21,8 \%$, com uma média de 19,2 consultas por turno, ressaltando a importância de uma boa estrutura física que garanta os dois consultórios por equipe na APS.

Com relação ao tipo de demanda, nessa equipe, eram realizados agendamentos de puericultura, pré-natal, tuberculose, acompanhamento de saúde mental e retornos. Apesar de apenas $13,5 \%$ dos registros serem de demandas programadas, ainda houve absenteísmo de $29,1 \%$ destes. Em um estudo realizado para descrever a prevalência de absenteísmo da agenda de atendimento médico de um serviço da APS também foi encontrada elevada prevalência e os autores recomendaram mudanças organizacionais para facilitar a adesão às consultas e sugeriram o modelo de AA para equilibrar a oferta e a demanda ${ }^{22}$. Além disso, no AA, o tempo decorrente das faltas das consultas programadas pode ser aproveitado para realizar 0 atendimento à demanda espontânea ${ }^{23}$.

Entre os usuários atendidos em demanda espontânea, apenas 2,8\% foram reagendados, sendo o restante, atendidos no mesmo dia, sem haver ociosidade para os profissionais devido ao absenteísmo dos usuários e garantindo o atendimento às necessidades do usuário no momento de sua procura. Há evidências de que o AA pode diminuir o tempo de espera entre a solicitação de um atendimento e a sua efetiva consulta, assim como a melhoria das taxas de absenteísmo, garantindo maior acessibilidade à população e diminuindo a probabilidade de os usuários buscarem outros meios de resolverem os seus problemas $^{2,9,10,24}$.

Pode-se observar que a maioria dos usuários chegava para atendimento nos primeiros horários dos turnos. Isso pode estar relacionado a aspectos culturais do antigo modelo de atendimento, onde caso os usuários não chegassem cedo, não conseguiriam ser atendidos pois o número de fichas era limitado ${ }^{25,26}$. Em outros estudos, alguns usuários relataram a espera de meses para conseguir um agendamento, e que pela necessidade, os mesmos encontravam soluções alternativas ao atendimento para a resolução dos seus problemas ${ }^{26}$. Os próprios usuários possuem falas relatando que se pudessem chegar a qualquer hora e serem atendidos seria bom ${ }^{25}$. 
Também pode-se observar um número elevado de usuários que buscam atendimento frequentemente na APS. É necessário identificar e caracterizar quem são esses usuários de forma a ter seu diagnóstico revisto e reavaliado ${ }^{27}$, de maneira a compartilhar as experiências, analisar os casos e planejar formas para lidar com tais situações ${ }^{28}$. Entender a demanda espontânea através dos MC, que é um dos elementos importantes da CIAP, fornece maior conhecimento sobre como e porque os usuários utilizam os serviços de saúde, o que possibilita a ampliação do acesso e uma resposta à demanda de maneira mais direcionada ${ }^{15}$.

A maioria dos usuários buscou atendimento devido a queixas ou sintomas (componente 1) seguido de diagnósticos definitivos (componente 7), que podem ser doenças crônicas ou a opinião deles sobre os seus sintomas, que são aceitos nos MC, mas que na avaliação do profissional a doença em questão pode não ser confirmada. Esses dados corroboram com um estudo realizado em Fortaleza-CE onde os mesmos componentes foram os mais encontrados ${ }^{17}$.

Observa-se um grande quantitativo do componente 4 (Resultado de exames) que foram atendidos em consultas individuais. O retorno de usuários com doenças crônicas para mostrar exames laboratoriais normais foi apontado por profissionais de uma ESF como um fator que contribui para a lotação das agendas. Para a resolução desse problema foi proposto que os resultados de exames normais fossem anotados no prontuário e os usuários fossem encaminhados para atendimentos em grupo. As consultas individuais seriam realizadas de acordo com a necessidade do usuário e não somente para mostrar exames. O mês de retorno do usuário também deve ser indicado de forma clara na receita e no prontuário para melhor organização ${ }^{29}$.

O capítulo A (Geral e não-específico) foi o mais encontrado nesse estudo e é o que engloba os principais MC presentes na literatura ${ }^{15,17,30}$, sendo utilizado para consultas relacionadas aos sistemas anatômicos múltiplos ou não-específicos ${ }^{13}$. Esse dado demonstra que na APS os problemas de saúde se apresentam de forma indiferenciada e, muitas vezes, não chegam a ser atribuídos a um diagnóstico específico ${ }^{15}$. É interessante ressaltar que apesar de os problemas sociais serem frequentes em territórios vulneráveis, eles não foram registrados por nenhum usuário como o $\mathrm{MC}$, evidenciando que os usuários podem não enxergar isso como um principal motivo para acessar o serviço.

A gravidez foi o segundo MC mais frequente entre o sexo feminino. Isso pode estar relacionado ao quantitativo de consultas segundo as recomendações do acompanhamento de pré-natal seguido pela equipe (mensal até a $28^{\circ}$ semana, quinzenal entre $28^{\circ}$ e $36^{\circ}$ semana e semanal após a $36^{\circ}$ semana), mas também, que a equipe precisa intensificar as ações de planejamento familiar para evitar casos de gravidez não planejada.

O terceiro motivo mais frequente entre as mulheres foi a busca pelo colpocitológico. Apesar de a agenda não ser programada para esse tipo de demanda com dia específico para ser realizado, há uma procura considerável das mulheres por esse procedimento. Também é importante destacar que muitas vezes elas relatavam como $\mathrm{MC}$ a realização do exame, mas que ao ser analisado pela equipe técnica, tratavase de uma queixa ginecológica e não a realização do procedimento. Sugerem-se estudos que comparem os MC com os diagnósticos após a avaliação pelo profissional técnico ou as principais demandas da APS identificadas em consultório.

Pode-se observar a diversidade das consultas realizadas pelo enfermeiro na APS, que estão relacionadas principalmente a saúde da mulher, criança e condições crônicas, mas que também estão 
relacionadas a $61 \mathrm{MC}$ diferentes. Existem evidências sobre o impacto do papel da enfermagem como prática avançada na melhoria da eficiência do sistema com qualidade do atendimento e melhora dos resultados na saúde dos usuários ${ }^{31}$. Com a organização da agenda e valorização da prática assistencial do enfermeiro os atendimentos de enfermagem contribuem para a ampliação do acesso na APS ${ }^{32}$. É importante que sejam revistas as metodologias de ensino visando diminuir a lacuna entre a formação e a prática dos profissionais de enfermagem, além do desenvolvimento de capacitações e protocolos específicos, objetivando a minimização de barreiras e à potencialização de facilitadores do processo ${ }^{33}$, assim como aumentar a quantidade de atendimentos direcionados a capítulos específicos da CIAP onde são identificados uma quantidade inferior de atendimentos quando comparado com a equipe médica.

\section{CONCLUSÃO}

Ao realizar o estudo da demanda nessa equipe, pode-se observar que o acesso avançado tem funcionado, mas há a necessidade de avaliações dos seus efeitos para que adequações possam ser realizadas.

A partir do estudo da demanda de uma equipe da ESF pode ser realizado um planejamento do processo de trabalho para que haja uma melhor organização da agenda e ampliação do acesso, refletindo em um melhor atendimento à população de forma resolutiva, oportuna e integral, com melhora das estratégias de promoção da saúde e prevenção de agravos, trabalhando a saúde em seu conceito ampliado, e indo além da queixa conduta.

A expansão da ESF impactou de maneira muito importante na saúde da população brasileira, com redução da mortalidade infantil e adulta relacionadas a algumas condições de saúde sensíveis à APSe a redução de hospitalizações. O acesso da APS pode ser ampliado aumentando o financiamento da ESF, reduzindo a população que é vinculada a cada equipe, diversificando as formas de comunicação com os usuários e investindo na formação de médicos de família e comunidade, com estímulo a sua interiorização e fixação, principalmente em áreas de maior vulnerabilidade.

Também deve-se explorar o trabalho em equipe, com a potencialidade clínica da enfermagem e a formação de enfermeiros especialistas em saúde da família. A diversidade dos atendimentos realizados pela enfermagem e o aumento da produtividade da equipe com a presença desse profissional nos mostra a importância do seu papel para a ampliação do acesso. É necessário investimentos na formação de enfermeiros especialistas em saúde da família para atuar nesse setor com sua prática baseada em protocolos e em concordância com os aspectos legais do exercício profissional.

Além disso, deve-se organizar e flexibilizar a agenda dos profissionais, para que todos os que procuram atendimento na APS possam ser acolhidos.

\section{Conflitos de interesse}

Declaram não haver conflitos de interesse.

\section{Contribuição dos autores}


Concepção e/ou delineamento do estudo: LEC, AFLS, JCACJ, NLPS. Aquisição, análise ou interpretação dos dados: LEC, AFLS, LSPP, JCACJ. Redação preliminar: LEC, AFLS, LSPP, JCACJ. Revisão crítica da versão preliminar: LEC, AFLS, NLPS. Todos os autores aprovaram a versão final e concordaram com prestar contas sobre todos os aspectos do trabalho.

\section{REFERÊNCIAS}

1. Ministério da Saúde. Portaria no 2.436, de 21 de setembro de 2017. Aprova a Política Nacional de Atenção Básica, estabelecendo a revisão de diretrizes para a organização da Atenção Básica, no âmbito do Sistema Único de Saúde (SUS). Diário oficial da União. Brasília, DF: 2017.

2. Ministério da Saúde. Portaria no 4.279, de 30 de dezembro de 2010. Estabelece diretrizes para a organização da Rede de Atenção à Saúde no âmbito do Sistema Único de Saúde (SUS). Diário oficial da União. Brasília, DF: 2010.

3. Murray M, Berwick D. Advanced access: reducing waiting and delays in primary care. JAMA 2003;289(8):1035-40. Estadunidense.

4. Murray M. Same-Day Appointments: Exploding the Access Paradigm. FPM 2000;7(8):45-50. Estadunidense.

5. Balasubramanian H, BiehI S, Dai L, Muriel A. Dynamic allocation of same-day requests in multi-physician primary care practices in the presence of prescheduled appointments. Health Care Manag Scl 2014;17(1):31-48. Estadunidense.

6. Starfield B. Atenção primária: equilíbrio entre necessidades de saúde, serviços e tecnologia. Brasília: UNESCO; 2002.

7. Costa ACM, Castro Júnior EF, Reis RS. O planejamento como instrumento de gestão. In: Costa AC, Pinho JRO. Processo de trabalho e planejamento em saúde. São Luís: EDUFMA; 2015. p. 21-32.

8. Rocha SA, Bocchi SCM, Godoy MF. Acesso aos cuidados primários de saúde: revisão integrativa. Physis (Rio J.) 2016;26(1):87-111.

9. Rivas J. Advanced Access Scheduling in Primary Care: A Synthesis of Evidence. J Healthc Manag. 2020;65(3):171-184.

10. Pires Filho LAS, Azevedo-Marques JM, Duarte NSM, Moscovici L. Acesso Avançado em uma Unidade de Saúde da Família do interior do estado de São Paulo: um relato de experiência. Saúde debate. 2019;43(121):605-613.

11. Brasil. Ministério da Saúde. Secretaria de Atenção à Saúde. Departamento de Atenção Básica. Cadernos de Atenção Básica: Acolhimento à demanda espontânea. Brasília; 2013; 1(28).

12. Costa ACM, Pinho JRO, Ferreira EB. O planejamento no processo de trabalho das equipes de saúde da família. In: Costa AC, Pinho JRO. Processo de trabalho e planejamento em saúde. São Luís: EDUFMA; 2015. p. 33-50.

13. World Organization of National Colleges, Academies, and Academic Associations of General Practitioners/Family Physicians. Classificação Internacional de Atenção Primária. 2 ed. Florianópolis: Sociedade Brasileira de Medicina de Família e Comunidade: 2009.

14. Guibu IA, Moraes JC, Guerra Junior AA, Costa EA, Acurcio FA, Costa KS, et al. Características principais dos usuários dos serviços de atenção primária à saúde no Brasil. Rev Saúde Pública 2017;51 Supl 2:17s.

15. Landsberg GAP, Savassi LCM, Sousa AB, Freitas JMR, Nascimento JLS, Azagra R. Análise de demanda em Medicina de Família no Brasil utilizando a Classificação Internacional de Atenção Primária. Ciênc Saúde Colet 2012;17(11):3025-36.

16. Pimentel IRS, Coelho BC, Lima JC, Ribeiro FG, Sampaio FPC, Pinheiro RP, et al. Caracterização da demanda em uma Unidade de Saúde da Família. Rev bras med fam comunidade. 2011;6(20):175-81.

17. Santos KPB, Ribeiro MTAM. Motivos de consulta mais comuns das pessoas atendidas por uma equipe de saúde da família em Fortaleza - CE. Rev Bras Med Fam Comunidade 2015;10(37):1-11.

18. Separavich MA, Canasqui AM. Saúde do homem e masculinidades na Política Nacional de Atenção Integral à Saúde do Homem: uma revisão bibliográfica. Saúde Soc 2013;22(2):415-28.

19. Gonçalves FC, Faria CCC. O acesso aos serviços de saúde: uma análise na perspectiva do gênero. Revista Perquirere 2016;13(1):13547.

20. Ximenes Neto FRG, Queiroz CA, Rocha J, Cunha ICKO. Por que eu não levo meu filho para a consulta de puericultura.... Rev. Soc. Bras. Enferm. Ped. 2010; 10(2):51-9.

21. Lima L. Estratégia de apoio às famílias para prevenção de internação pediátrica por condições sensíveis à Atenção Primária à Saúde. Botucatu. Dissertação [Mestrado] - Universidade Estadual Paulista “Júlio de Mesquita Filho”, Faculdade de Medicina de Botucatu. 2018. 
22. Silveira GS, Ferreira PR, Silveira DS, Siqueira FCV. Prevalência de absenteísmo em consultas médicas em unidade básica de saúde do sul do Brasil. Rev Bras Med Fam Comunidade. 2018;13(40):1-7.

23. Cirino, FMSB, Schneider Filho, DA, Nichiata, LYI, Fracolli, LA. O Acesso Avançado como estratégia de organização da agenda e de ampliação do acesso em uma Unidade Básica de Saúde de Estratégia Saúde da Família, município de Diadema, São Paulo. Rev Bras Med Fam Comunidade. 2020;15(42):1-11.

24. Ansell D, Crispo JAG, Simard B, Bjerre LM. Interventions to reduce wait times for primary care appointments: a systematic review. BMC Health Serv Res. 2017;17(1):1-9.

25. Lima SAV, Silva MRF, Carvalho EMF, Cesse EAP, Brito ESV, Braga JPR. Elementos que influenciam o acesso à atenção primária na perspectiva dos profissionais e dos usuários de uma rede de serviços de saúde do Recife. Physis (Rio J.). 2015;25(2):635-56.

26. Marin MJS, Moracvick MYAD, Marchioli M. Acesso aos serviços de saúde: comparação da visão de profissionais e usuários da atenção básica. Rev enferm UERJ. 2014;22(5):629-36.

27. Carvalho IPA, Carvalho CGX, Lopes JMC. Prevalência de hiperutilizadores de serviços de saúde com histórico positivo para depressão em Atenção Primária à Saúde. Rev Bras Med Fam Comunidade. 2015;10(34):1-7.

28. Zoboli ELCP, Santos DV, Schveitzer MC. Pacientes difíceis na atenção primária à saúde: entre o cuidado e o ordenamento. Interface (Botucatu). 2016;20(59):893-903.

29. Reigada, CLL. Repensando acesso, qualificando o cuidado: relato do trabalho em duas equipes de saúde da família. Rev Bras Med Fam Comunidade. 2017;12(39):1-7.

30. Gusso GDF. Classificação Internacional de Atenção Primária (CIAP 2). São Paulo. Tese [Doutorado]. Faculdade de Medicina da Universidade de São Paulo; 2009.

31. Bryant-Lukosius D, Valaitis R, Martin-Misener R, Donald F, Peña LM, Brousseau L. Enfermagem com prática avançada: uma estratégia para atingir cobertura universal de saúde e acesso universal à saúde. Rev. Latino-Am. Enfermagem. 2017;25:e2826.

32. Lowen IMV, Peres AM, Ros C, Poli Neto P, Faoro NT. Inovação na prática assistencial do enfermeiro: ampliação do acesso na atenção primária. Rev Bras Enferm. 2017;70(5):898-903.

33. Ribeiro GC, Padoveze MC. Sistematização da Assistência de Enfermagem em unidade básica de saúde: percepção da equipe de enfermagem. Rev Esc Enferm USP. 2018;52:e03375. 